\title{
Convivencia escolar antes y durante la pandemia. Análisis de la experiencia escolar y las prácticas de participación desde las voces del estudiantado
}

\section{School harmony before and during the pandemic. Analysis of the school experience and participation practices from the students' voices}

\section{LuCÍA LITICHEVER*}

DENISE LAURA FRIDMAN ${ }^{* *}$

El artículo analiza los diferentes modos de implementación del sistema de convivencia en escuelas secundarias de gestión estatal y privada de la Ciudad de Buenos Aires, Argentina, desde la perspectiva del alumnado. La metodología del estudio fue mixta: se aplicó una encuesta en línea y se realizaron entrevistas y grupos focales. En la indagación se abordaron aspectos de la convivencia previos a la pandemia y nuevas situaciones surgidas durante la educación a distancia. Se encontró que los reglamentos de convivencia en las escuelas son la norma común, cuya aplicación es considerada por los estudiantes discrecional, por lo que apelan a una mayor participación en el proceso de elaboración de las reglas y el diálogo como modo de resolución de los conflictos de convivencia. Se observa que durante la educación a distancia en el contexto de la pandemia se han fortalecido los vínculos entre estudiantes y los conflictos más recurrentes están relacionados con la sobrecarga académica.

The article analyzes the implementation modes of the harmony system within public and private high schools in the City of Buenos Aires, Argentina, from the students' perspective. A mixed methodology was combined by applying an online survey, interviews and focus groups. The research addressed living aspects prior to the pandemic and the new situations that arose during distance education. Students expressed disparity in the schools' rules use -often discretionary as a common norm-and sought participation in the rule's development process and dialogue to resolve conflicts. It is observed that during distance education in the context of the pandemic, the links between students have strengthened and the most recurrent conflicts relate to academic overload.

\author{
Palabras clave: \\ convivencia \\ escolar, escuela \\ secundaria, \\ estudiantes, \\ sanciones, \\ pandemia
}

\section{Keywords:}

school harmony, high school, students, sanctions, pandemic

Recibido: 29 de diciembre de 2021| Aceptado para su publicación: 28 de julio de 2021|

Publicado: 6 de septiembre de 2021

Cómo citar: Litichever, L. y Fridman, D. L. (2021). Convivencia escolar antes y durante la pandemia. Análisis de la experiencia escolar y las prácticas de participación desde las voces del estudiantado. Sinéctica, Revista Electrónica de Educación, (57), e1248. https://doi.org/10.31391/ S2007-7033(2021)0057-010

\footnotetext{
* Magíster en Ciencias Sociales con orientación en Educación por Flacso Argentina. Investigadora del Área de Educación de esta misma institución, donde codirige el Núcleo de Estudios sobre la Escuela y los Vínculos Intergeneracionales. Líneas de investigación: educación secundaria, convivencia, participación juvenil y desigualdad. Correo electrónico: lucialitichever@gmail.com

** Magíster en Problemáticas Infanto Juveniles por la Universidad de Buenos Aires. Docente y responsable del Programa de Género y Diversidad Sexual en la Universidad Pedgógica Nacional e investigadora del Área de Educación en Flacso. Líneas de investigación: convivencia escolar y resolución de conflictos en la escuela secundaria. Correo electrónico: dlfridman@gmail.com
} 


\section{INTRODUCCIÓN}

$7 \mathrm{n}$ la Argentina, a finales del siglo pasado, se inició un proceso de revisión de los regímenes de disciplina de las escuelas secundarias para el desarrollo de sistemas de regulación de los vínculos en pos de promover modos más democráticos e inclusivos. La Ciudad de Buenos Aires fue impulsora en este sentido, al avanzar con mayor prontitud que otras jurisdicciones en la revisión, análisis y consulta de estos mecanismos y nuevas propuestas. En este marco se establece, en 1999, la Ley № 223 del Sistema Escolar de Convivencia. Hoy, a más de dos décadas de su promulgación, resulta pertinente analizar su implementación, los cambios generados en las instituciones a partir de dicha ley, así como la diversidad de situaciones en los distintos establecimientos.

En este año tan particular, en el que la pandemia mundial producto de la COVID-19 llevó a muchos países a emprender políticas de aislamiento, en la Argentina se decretó el 19 de marzo de 2020 el aislamiento social preventivo y obligatorio (ASPO-DECNU-2020-297-APN-PTE-Disposiciones, publicado en el Boletín Oficial el 20 de marzo de ese año), a partir del cual se tomó la decisión del cierre de los establecimientos educativos y la continuidad de la escolaridad a través de la educación a distancia.

Nuestro equipo de investigación tenía previsto realizar el trabajo de campo en las escuelas, pero ante la situación de aislamiento nos vimos en la necesidad de repensar nuestro abordaje (nuestro modo de indagación y acercamiento a las escuelas) y surgieron preguntas asociadas al nuevo contexto: ¿qué ocurre con la convivencia en esta nueva situación?, ¿aparecen nuevos conflictos?, ¿cómo se resuelven?, ¿qué regulaciones se mantienen?, ¿qué nuevas formas toman los vínculos intra- e intergeneracionales? También nos pareció que resultaba un momento propicio para conversar sobre la convivencia en las escuelas y tomar la distancia que nos permitía esta situación tan particular. Así, reformulamos nuestras maneras habituales de efectuar las indagaciones y conformar la muestra de modo que hiciera posible la investigación en este contexto con nuevas preguntas.

Dussel (2005) plantea que la escuela constituye "un campo de experiencias en el cual se aprenden ideas y prácticas sobre la legalidad, la legitimidad, la justicia, la construcción de un orden social y la resolución de conflictos, y en ese sentido da indicios de procesos más generales que tienen lugar en la sociedad" (p. 100). Así, una manera de analizar la formación política, las relaciones de poder y las desigualdades es indagar el orden disciplinario que la escuela instaura.

Pensar en estos términos es considerar que la política no se presenta como un don natural, sino que necesita instancias de aprendizaje y producción (Kriger y Dukuen, 2017). A pesar de las crisis históricas de las instituciones modernas, la escuela y la familia aún conservan un lugar importante como agentes de esa socialización (Berger y Luckmann, 1986). Este proceso debe ser comprendido en el marco de un contexto general e institucional que permita identificar sus características. En el ámbito educativo no podemos dejar de soslayar que las condiciones para la socialización política son atravesadas por relaciones de poder desiguales entre el mundo adulto y los jóvenes que legitiman ciertas prácticas más tradicionales frente a otras que pugnan por crear un marco de justicia e inclusión. 
Las disputas que se dan en torno a los conflictos de convivencia escolar y su tratamiento constituyen un aspecto central de la socialización política de los jóvenes. En este proceso dinámico, complejo y situado, el conflicto es reconocido como un factor integrador, ya que es una forma de socialización necesaria (Simmel, 1908/2010). Esta última es pensada como creadora de comunidad donde el conflicto, en cuanto relación, es identificado como elemento constitutivo de la cohesión social, contra todo pensamiento de sentido común que lo ubica como un elemento de resquebrajamiento.

Por su parte, Mouffe (2005) señala que el conflicto no debería erradicarse dado que la especificidad de la democracia moderna reside en su reconocimiento y legitimación. De esta forma, "lo que la política democrática requiere es que los otros no sean vistos como enemigos a ser destruidos sino como adversarios cuyas ideas serán combatidas, incluso de modo virulento, pero cuyo derecho a defenderlas nunca será puesto en cuestión" (Mouffe, 2005, p. 86).

En el ámbito escolar, los conflictos ponen de manifiesto diferencias de variada índole, que expresan la tensión propia de un espacio social donde interactúan personas con roles, trayectorias, necesidades, contextos y culturas disímiles (Siede, 2007). La convivencia de estudiantes y docentes con diferencias de género, clase, estilos, como lo menciona Viscardi (2008), requiere reconocer al otro como igual a uno mismo o como miembro de una misma comunidad en el sentido de reconocerle atributos similares a los propios. Ser integrante de una comunidad se constituye a partir de la creencia de que estamos "sometidos a las mismas normas y a formas de vida y valores, si no iguales, al menos respetables y compatibles; $y$, sobre todo, titular de unos derechos y sujeto de unas obligaciones recíprocas" (Fernández, 2012, p. 19).

Siede (2007) indica que es importante cuidar que estas responsabilidades y derechos se enmarquen en dos principios: el de legalidad, que postula que ningún miembro de la comunidad escolar se le pueden reconocer menos derechos que los que la ley le otorga fuera de la escuela, y el principio pedagógico, a partir del cual no se puede adjudicar a los estudiantes mayor grado de responsabilidad que el que se espera de un ciudadano adulto en la sociedad. Así, estos principios, establecen un piso de derechos y un techo de responsabilidades en los que debe circunscribirse la participación de los estudiantes.

La escuela tiene como una de sus funciones la formación y promoción de prácticas ciudadanas, que respetan las identidades particulares del estudiantado para que, a su vez, puedan aportar a la construcción de una universal que permita convivir en sociedad (Tenti, 2009). Esta formación ciudadana abarca tres aspectos fundamentales. Por una parte, desde un plano más general, se relaciona con la perspectiva de los derechos humanos de los niños, niñas y adolescentes presentes en el ámbito escolar. El segundo refiere la conformación de espacios de participación democráticos y la normativa vinculada a la regulación de la convivencia. El tercero ubica la formación ciudadana en espacios curriculares específicos para ello (Viscardi y Alonso, 2013).

En relación con los dos primeros aspectos, la definición y puesta en práctica de las normas cobra un lugar central. Permite delinear un espacio común que busca preservar a todos y todas. Esto supone, como lo plantea Southwell (2020), que siempre se mantenga abierta la pregunta acerca de su legitimidad, sus condiciones y sus 
efectos, ya que ahí reside su potencialidad democratizadora. A su vez, estas normas permiten al alumnado sentirse parte de una comunidad política siempre y cuando la escuela sea "capaz de transmitirle[s] en todo momento, a través de una organización eficaz de la convivencia cotidiana y de una labor permanente de tutela individual y colectiva por parte de los profesores, que ya es[son] parte de una comunidad educativa" (Fernández, 2012, p. 26).

Otro principio que resulta útil para la elaboración de las normas, según Siede (2007), es la distinción entre legalidad y arbitrariedad; si la norma es arbitraria, está definida por la autoridad; en cambio, si la norma es necesaria, está definida por su legitimidad y, así, se ubicaría en el centro el derecho o la obligación para el colectivo y no para unos sujetos en particular.

Siede (2007) señala que las normas requieren la previsión de sanciones o algún tipo de consecuencias para quienes las violan. El autor plantea que el ejercicio responsable de la autoridad en la regulación de la vida social escolar implica "volver a dar sentido a las sanciones, con una finalidad democrática y transformadora" (p. 191).

A lo largo de este escrito, buscamos poner el foco en dos elementos vinculados: las interacciones cotidianas y los aspectos que pretenden definir el conjunto de reglas y pautas que se establecen en las escuelas. En ambas dimensiones podemos encontrar desigualdades basadas en diferencias generacionales que provocan dificultades en el vínculo docente-alumno/a, conflictos de género que aparecen en las expectativas diferenciadas para los estudiantes varones y mujeres, así como otros tipos de conflictividades.

La indagación nos permite analizar la consolidación de la convivencia como temática abordada en las instituciones educativas a lo largo de estas décadas y nos advierte sobre la disparidad de criterios a la hora de aplicar la norma o sancionar frente a las transgresiones que se presentan. A su vez, nos introduce en los conflictos suscitados en este contexto inédito de convivencia virtual.

\section{MÉTODO}

\section{Diseño}

La pesquisa se llevó a cabo a partir de una metodología de carácter mixto, que combina técnicas cuantitativas y cualitativas. El objetivo de la investigación fue indagar y analizar la experiencia educativa de jóvenes varones y mujeres a partir de tres dimensiones: los modos en que dan sentido a su experiencia de escolarización actual, pasada y futura; las prácticas de participación; y las vinculadas al uso de tecnologías. Para ello, nos propusimos realizar una descripción de la complejidad que organiza la trama de relaciones en las escuelas secundarias y la posibilidad de inscribir estas en relaciones de mayor extensión que permitan una imbricación teórica-empírica (Rockwell, 2009). En este artículo nos circunscribimos al análisis de la experiencia escolar y las prácticas de participación mediante la recuperación de las voces del estudiantado. 


\section{Participantes}

En junio y julio de 2020 aplicamos una encuesta virtual en la que participaron 626 jóvenes que se encontraban cursando cuarto, quinto o sexto año, con un rango etario de entre 15 y 18 años de diferentes escuelas de la Ciudad de Buenos Aires. De los encuestados, se destaca la pareja distribución de respuestas entre el alumnado de escuelas de gestión estatal (59\%) y de gestión privada (41\%), cuando esta última suele ser una población de más difícil acceso para las investigaciones. También destaca la variedad de escuelas de pertenencia de los estudiantes varones y mujeres que respondieron la encuesta, que llegaron a un total de 107 escuelas: algunas dependientes de la Universidad de Buenos Aires y otras de gestión estatal, de modalidades bachiller, comercial y técnica; otras privadas, entre las cuales hay una gran diversidad de escuelas laicas o religiosas de distintos cultos.

Según el Anuario Estadístico Educativo 2019 (Ministerio de Educación, 2020), en la Ciudad de Buenos hay 496 unidades educativas de nivel secundario común, entre las que se incluyen las 107 de la encuesta. De este total de unidades educativas, 157 son del sector estatal y 339, del sector privado, lo que marca una característica notable de esta jurisdicción: la preponderancia del sector privado, que la distingue de otras jurisdicciones del país. La encuesta fue anónima e incluyó, para quienes quisieran, al final de ella, un espacio abierto para dejar un medio de comunicación para participar en entrevistas y grupos focales. No buscamos alcanzar una muestra por cuotas de escuelas, sino llegar a la mayor cantidad de jóvenes a través del método de bola de nieve.

A diferencia de otras investigaciones que este mismo grupo venía realizando, en las cuales el contacto con los estudiantes se daba en las escuelas, el contexto del aislamiento social preventivo y obligatorio implicó que la aproximación al universo fuera individual y no institucional. Por eso, la encuesta se compartió en redes sociales, con docentes, preceptores, autoridades de escuelas, actores vinculados a la gestión educativa, comisiones de centros de estudiantes, y familiares y conocidos que la enviaron a estudiantes que, a la vez, la compartieron con otros compañeros y compañeras.

Organizamos cinco grupos focales de manera remota entre septiembre y diciembre de 2020; en cada uno participaron entre cuatro y nueve estudiantes mujeres y varones de distintas escuelas. Además, aplicamos entrevistas individuales a 12 estudiantes de diferentes escuelas a fin de cubrir la diversidad de tipos de establecimientos. Consideramos como unidad de análisis la experiencia de estudiantes de escuelas secundarias en distintas instituciones. Para la elaboración de los grupos focales, conformamos una muestra intencional no probabilística, en la que priorizamos como criterio de selección el tipo de institución: escuelas de circuitos de élite económica o académica (de gestión privada e instituciones dependientes de la universidad), escuelas de gestión privada confesionales, escuelas técnicas de gestión estatal, escuelas bachiller o comercial de gestión estatal.

Para la participación en los grupos focales y las entrevistas, cada estudiante, o su adulto/a responsable en caso de ser menor de edad, completó un consentimiento que constaba que la información recabada solo sería utilizada para la investigación y que se respetaría siempre el anonimato de los participantes. Los estudiantes que han participado en el estudio pertenecen al contexto urbano de 
la capital del país, muy distinto al que puede ser el de las pequeñas ciudades o el medio rural, de ahí que sus respuestas y el análisis deben ser interpretados desde ese marco, sin pretender ser extensivas a otras realidades. De todos modos, resultan pertinentes para pensar los vínculos y sus experiencias en las instituciones educativas de nivel secundario.

\section{Técnicas de producción}

La encuesta se administró de manera virtual a través de un formulario de Google y se llevó a cabo una prueba piloto del formulario (en su formato, claridad de preguntas, variables de respuesta, tiempo de resolución, accesibilidad, facilidad de respuestas y visibilidad en distintos aparatos electrónicos: celulares, tabletas y computadoras). Esta primera instancia fue realizada con ocho estudiantes de distintas escuelas que contestaron la encuesta mientras conversaban por videollamada con investigadores del equipo, quienes registraban el modo y la actitud de los estudiantes varones y mujeres al responder y que luego conversaron sobre la claridad del instrumento.

Tanto la encuesta como las entrevistas y los grupos focales incluyeron preguntas referidas a la escolaridad en tiempos de educación a distancia producto del confinamiento por la COVID-19 y otras que proponían temáticas sobre la escolaridad presencial previa. En el caso de los grupos focales, el eje principal fue la convivencia escolar, en el cual se trabajó a partir de casos hipotéticos que permitieran conocer los modos de resolución de conflictos en cada una de sus escuelas, así como sus percepciones al respecto. Además, podemos inferir que, a diferencia de investigaciones precedentes, la particularidad de la implementación de esta indagación en el contexto de la pandemia, en el que el diálogo con los jóvenes se desarrolló en el ámbito de sus hogares, ayudó a generar un espacio que habilitó un intercambio más llano, sin condicionamientos institucionales.

\section{DESARROLLO}

Cada escuela es un mundo: una norma común, disparidad de situaciones

Como presentamos en la introducción, la Ciudad de Buenos Aires cuenta desde 1999 con la Ley № 223, que prescribe la conformación del sistema de convivencia en las escuelas. Esta normativa se propone

\footnotetext{
propiciar la participación democrática de todos los sectores de la comunidad educativa, según las competencias y responsabilidades de cada uno, en la elaboración, construcción y respeto de las normas que rijan la convivencia institucional con el fin de facilitar un clima de trabajo armónico para el desarrollo de la tarea pedagógica (Ciudad de Buenos Aires, 1999, artículo 6ํㅜ).
}

Asimismo, describe los principios, objetivos y valores que deben ser observados, cumplidos y promovidos por el Sistema Escolar de Convivencia. Determina los criterios que deben respetarse en la aplicación de las normas de convivencia, y fija las sanciones y los modos de aplicación. Establece que las normas deben ser contextualizadas para respetar la identidad de cada comunidad educativa. 
La normativa también especifica que cada institución debe redactar su propio reglamento de convivencia y organizar su consejo de convivencia. Esto último es de carácter obligatorio en el caso de las escuelas de gestión estatal y optativo para las escuelas de gestión privada, que durante el tratamiento legislativo ejercieron presión para lograr que su autonomía político-educativa quedara incluida en el texto final (Fridman, 2014).

A pesar de esta diferencia, la ley se presenta como un marco común en la regulación de las relaciones al interior de las instituciones educativas de la jurisdicción a la vez que habilita y promueve el abordaje específico de cada escuela en función de sus propias características. Si bien establece pautas para el reglamento y el funcionamiento del consejo, deja un amplio margen de decisión para las normas que se establecen, la manera en la que se les enuncia, los actores que están sujetos a ellas, así como el modo de funcionamiento de los consejos, sus funciones y las formas de resolución de los conflictos.

En nuestras indagaciones (Litichever et al., 2008, 2012, 2019; Fridman y Litichever, 2016; Southwell, 2020, entre otras) hallamos que no todas las escuelas han implementado estos dispositivos. Si bien la mayoría cuenta con reglamentos de convivencia y los distintos actores saben de su existencia, su contenido no siempre es conocido, tal como lo enuncian los estudiantes en las citas que incluimos.

A veces desconocen quiénes los han redactado y dejan en claro la escasa o nula participación del cuerpo estudiantil, o expresan que no se recurre a ellos para la resolución de las situaciones problemáticas. En los grupos focales y entrevistas realizadas así lo manifiestan los estudiantes: "Las normas, si no me equivoco, están. Cada uno tiene el cuaderno de comunicaciones y están ahí al principio. Lo armó la escuela" (estudiante varón, 5to año, escuela privada laica); "Alguna vez suspendieron a alguien un par de días, pero no sé qué tenés que hacer hasta llegar ahí, hay un montón de normativa que está escrita y por ahí ni sabes que existe" (estudiante mujer, 5 to año, escuela privada religiosa).

Por su parte, los estudiantes de escuelas estatales parece que conocen la normativa vigente y buscan modificar los aspectos con los que desacuerdan: "Todos los años estamos tratando de modificar o mejorar el código de vestimenta, pero las reglas no las hablamos nunca" (estudiante varón, 4to año, escuela estatal técnica):

En nuestro colegio se logró derogar el código de vestimenta, se dejaron sólo las ojotas [hace referencia a chanclas, sandalias de goma], las partes que implican un riesgo para les chiques [utilización del lenguaje inclusivo que refiere a varones, mujeres y personas de la diversidad sexual], pero se quitaron los shorts, polleras, remeras, sin embargo hay preceptores que siguen insistiendo con eso y hay mucho conflicto por esas razones (estudiante mujer, 5to año, escuela estatal bachiller).

Las voces de estos jóvenes nos permiten identificar que, a diferencia de lo que ocurría en años anteriores, hoy a más de dos décadas de la implementación de la ley de convivencia, aquellos conocen la existencia de reglamentos de convivencia en sus escuelas, aunque como lo expresan, algunos saben que están, pero no conocen bien sus normas y otros las conocen y pugnan por generar modificaciones; en este sentido, demandan una participación más activa en su elaboración y que se consideren sus pareceres. Esto último ocurre de forma más sostenida en las escuelas de gestión estatal, donde la legislación, como mencionamos, tuvo mayor presencia. 
En este punto, es interesante revisar la respuesta del alumnado ante dos preguntas de la encuesta: su percepción respecto de la adecuación de las normas en sus escuelas y su deseo de participación en la redacción de estas. En relación con la primera cuestión, casi el 50\% considera que las normas de convivencia son adecuadas, lo que nos habla de su pertinencia para la mitad de quienes respondieron la encuesta; no obstante, no hay que perder de vista que una tercera parte de ellos (36.9\%) plantea estar ni de acuerdo ni en desacuerdo con ellas; se encuentran en un punto medio, por lo que podríamos inferir que les da lo mismo, que no las conocen o que no se ponen de manifiesto con frecuencia, por lo cual no pueden evaluar su pertinencia.

Asimismo, encontramos una porción (15\%) de estudiantes que consideran que las normas de su escuela no son adecuadas para la regulación de la convivencia en el cotidiano escolar. En las entrevistas y grupos focales, los estudiantes nos permiten visualizar algunas razones de esta inadecuación: por considerarlas injustas, arbitrarias y, otras veces, por no encontrarles un sentido. Durante un grupo focal se estableció un diálogo interesante al respecto entre dos estudiantes de una misma escuela:

Estudiante 1: Hay reglas que no tienen mucho sentido, pero que nadie cumple, como que no se pueden sacar fotos [...] algunas cosas como que sorprenden porque no tienen mucho sentido (estudiante varón, 4to. año, escuela privada laica).

Estudiante 2: Pará, a mí me pusieron una amonestación por eso [...] Después con el Centro de Estudiantes aprendí que no me pueden hacer eso, pero en ese momento fue como que prefería no pelear o tenía miedo de que me pongan otra amonestación más (estudiante mujer, 5to. año, escuela privada laica).

Otra estudiante agregó: "Y además hay restricciones como no podés llevar un piercing o aros grandes y me sorprendió porque yo debería poder llevar un piercing si quiero. Nadie anda corriendo por el pasillo para que sea peligroso" (estudiante mujer, 4to. año, escuela privada laica).

La segunda pregunta resulta aún más contundente, los estudiantes sostienen con una amplia mayoría (81\%) que deberían participar en la decisión de las normas escolares. Ahí se pone de manifiesto su deseo de ser protagonistas, de sumar sus voces y opiniones al quehacer escolar, y poder ser partícipes de esta instancia, asunto que en la ley se define con claridad, pero que todavía cuesta que se lleve adelante en muchos establecimientos.

Aquí es interesante recuperar que durante el debate legislativo para la sanción de la Ley № 223, el grado y el modo de participación de los estudiantes fue uno de los aspectos más controversiales. Las posiciones contrapuestas sostenían, por un lado, que el involucramiento del estudiantado en la conformación de las normas y las decisiones sobre su aplicación implicaba una pérdida de autoridad para los docentes, mientras que otras posturas abonaban a la importancia de habilitar ese lugar en pos de construir escuelas que promuevan prácticas democráticas (Fridman, 2014).

Esta posibilidad para que los estudiantes se involucren rompe con algunas estructuras duras de la escuela, ya que propone un ámbito de discusión colegiada en un espacio que, históricamente, se constituyó como jerárquico y verticalista (Litichever, 2019). Paulín (2002) señala que la discusión colegiada establece formas distintas de circulación del poder y espacios de autoridad diferentes a los históricos escolares 
enmarcados en roles unipersonales del director y el profesor, y que este cambio en la oportunidad de tomar decisiones es muchas veces significado por los docentes como una restricción de su poder y su rol.

Esta situación conduce, en ocasiones, a que se termine obstaculizando la puesta en marcha de estas instancias de participación (Litichever, 2019). Esto pone en evidencia la búsqueda por sostener posiciones desiguales de poder de las personas adultas por sobre las jóvenes. Sin embargo, la intervención de estas últimas es fundamental e implica darles la posibilidad de participar en decisiones de cuestiones que las involucran. A su vez, como lo señala Tenti (2009), es necesario que el estudiantado pase de un lugar receptor de la norma a una instancia de productores y sea partícipe del análisis de los casos en los que se requiere su aplicación, de forma que vaya construyendo su autonomía.

En algunas escuelas, este deseo de participación en la redacción de las normas constituyó un proceso de organización y manifestación para promover la derogación de algunos aspectos, sobre todo los vinculados al código de vestimenta. Así, como se enuncia en el verbatim citado, en ocasiones lograron producir cambios, aunque algunas personas adultas parecen no haberlos aceptado y siguen insistiendo en que respeten aspectos del código ya derogados. En otras escuelas obtuvieron pequeñas modificaciones o siguen pugnando por conseguirlas: "En mi escuela se logró incorporar la bermuda, pero sólo para los meses de verano porque nos moríamos de calor" (estudiante varón 5to año, escuela estatal técnica). Por su parte, otro estudiante también de escuela técnica comenta que, luego de mucha pelea, lograron cambios más radicales: "En mi escuela se logró desestimar el código de vestimenta y se dejaron reglas sólo para el espacio de taller porque conlleva un riesgo" (estudiante varón 4to, escuela estatal técnica). A su vez, una estudiante de una escuela dependiente de la universidad señala:

Las chicas no podían ir con short y los pibes sí podían ir en bermudas entonces se hicieron un par de shortazos [hace referencia a la organización de una jornada escolar en la que todo el estudiantado concurre con short para manifestarse en contra de la prohibición de su uso] y logramos modificarlo. Los profesores antes hacían algunos comentarios y tuvieron que dejar de decir esas cosas (estudiante mujer, 5to año, escuela estatal dependiente de universidad nacional).

La modificación del código de vestimenta ha sido una bandera para el cuerpo estudiantil de las escuelas secundarias en la Argentina en los últimos años como un modo de resistencia a las regulaciones del cuerpo atravesadas por desigualdades de género. A pesar de que este tema excede el contenido del artículo, no podemos dejar de mencionar la resonancia que ha tenido el movimiento feminista en los vínculos al interior de las escuelas secundarias en los últimos años, como es el caso de la regulación de la ropa, que busca modificar relaciones desiguales entre varones, mujeres y personas de la diversidad sexual (González, 2017).

Los datos de la encuesta y las opiniones del estudiantado en las entrevistas y los grupos focales nos muestran que tienen mucho para decir respecto a su vivencia en la escuela y las normas que los regulan. Dar lugar a las voces estudiantiles se vuelve imperioso para crear un espacio de encuentro y un tránsito por la escolaridad más democrático y que posibilite ir construyendo mayor autonomía en su socialización política. 


\section{SANCIONES ESCOLARES: DESDE EL APERCIBIMIENTO AL DIÁLOGO}

Como señalamos, otra de las instancias que propone la ley de convivencia es la organización de consejos de convivencia. Encontramos que son pocas las escuelas que los han conformado y, donde se les convoca, funcionan como entes pedagógicos en la resolución de los conflictos. Si bien la normativa incluye a todos los establecimientos de la jurisdicción, hay una amplia disparidad que se acrecienta aún más según la gestión de dependencia de las instituciones. La ley incluye esta modalidad como obligatoria en los planteles de gestión estatal, pero no en los de gestión privada o en las escuelas dependientes de la universidad.

Esta diferencia puede apreciarse en el grado de acuerdo manifestado en la encuesta por los estudiantes con la frase "En mi escuela convocan al Consejo de Convivencia para resolver los conflictos". Los estudiantes que están de acuerdo con la frase se concentran en las escuelas de gestión estatal no universitarias con un 57\%; este valor disminuye a un $26 \%$ para estudiantes que concurren a escuelas de gestión estatal dependientes de una universidad nacional y baja a un $17 \%$ para quienes asisten a secundarias de gestión privada. De modo inverso, el mayor porcentaje de respuestas en desacuerdo con la frase se agrupa entre quienes estudian en estas últimas instituciones, con un 69\%, porcentaje que disminuye a $19 \%$ de respuestas de estudiantes de escuelas de gestión estatal y a $17 \%$ entre quienes pertenecen a establecimientos dependientes de una universidad nacional. Estas opiniones permiten afirmar que, en los casos en los que la puesta en marcha de los consejos no es obligatoria, no se les ha considerado como una opción para resolver los conflictos.

Para conocer un poco más acerca del funcionamiento del consejo en las escuelas donde fue conformado, es interesante complementar estas respuestas con las intervenciones en los grupos focales y entrevistas que nos ayudan a comprender que, en muchos casos, el alumnado no conoce qué es el consejo de convivencia y, salvo que ellos o ellas o algún compañero o compañera cercano haya concurrido a él, desconocen si se ha convocado, cuándo y cuántas veces. Podríamos inferir de ello que la presencia del consejo de convivencia en las escuelas aún no tiene el nivel de injerencia o tipo de funcionamiento que la ley plantea.

El consejo de convivencia tiene como una de sus funciones asesorar al rector/a en la sanción que se aplica en una situación de transgresión grave a una norma escolar, además de convocar para trabajar cuestiones vinculadas a la prevención de conflictos, la redacción del reglamento de convivencia o abordar alguna temática específica de la escuela. Para eso, se integra de representantes de los diferentes claustros escolares que, ante una falta grave, garantizan el derecho a ser oído/a de todas las partes involucradas. Desde esta modalidad, el consejo de convivencia cobra un lugar importante en la escuela al promover los derechos del estudiantado que ha transgredido una norma desde una perspectiva de gestión del conflicto, que, muchas veces, no llega a una sanción, sino más bien a establecerse como un lugar de escucha y contención. Cuando se convoca es un espacio valorado; un estudiante comenta al respecto:

Sí, hay consejo de convivencia y está bueno, es una instancia que se aprovecha mucho, sobre todo para los pibes. Tuvimos varios casos, uno muy fuerte de un pibe que empujó a la vicerrectora. Complicado ver cómo hacer para que no lo echen, la vicerrectora no lo quería echar, pero hay una cuestión del marco legal institucional que hay cosas que no 
podés pasar por alto. Tuvimos varios consejos de convivencia. Funcionan bien porque se buscan otras estrategias. A esa población de la escuela secundaria si vos la echás, no vuelve nunca más al colegio (estudiante varón, 5to. año, escuela estatal bachiller).

Otro estudiante refiere que en su escuela se convoca al consejo de convivencia, pero cuestiona sus decisiones: "En el mío [refiriéndose a su colegio] se hace consejo de convivencia, pero la gran mayoría de los casos siempre terminan en una sanción y una suspensión en vez de contener al pibe" (estudiante varón, 5to. año, escuela estatal bachiller).

En las entrevistas y los grupos focales, los estudiantes varones y mujeres mencionan las sanciones escritas y las separaciones transitorias como los modos más comunes de actuar por parte de la escuela ante una falta sin apelar tanto a formas colectivas de gestionar el conflicto, como podría ser con la intervención de los consejos. Unas y otras instancias responden a diferentes modelos institucionales que reconocen el conflicto en el ámbito escolar según se ubiquen desde un plano individual (modelo hermenéutico interpretativo) y tienen como respuesta una sanción escrita o suspensión o pretenden una mirada del conflicto como un aspecto constructivo (modelo crítico) y, por ende, buscan su gestión desde un abordaje democrático y participativo (Jares, 1997). Tomar esta mirada sobre el conflicto como gestión nos parece fundamental, dado que introduce la perspectiva colectiva por sobre las acciones individuales.

Jares (1997) propone un tercer modelo (tecnocrático positivista) vinculado al abordaje de los conflictos como aquel que los percibe como patológicos y, por ende, los niega. En las voces del estudiantado se pone de manifiesto que, en ocasiones, en sus escuelas ocurre esta negación de la situación conflictiva, lo que da como resultado su no intervención. Un ejemplo de ello se produce cuando, frente al modo de resolución individual o de negación que se aleja de la posibilidad de un abordaje más cabal del conflicto y ante la falta de intervención institucional respecto de situaciones que aquejan a las estudiantes (sobre todo) por eventos de acoso, comenzó a hacerse frecuente en varias escuelas la modalidad escrache, que consiste en señalar públicamente a quien está teniendo una actitud o realizando una acción que molesta a otras (Faur, 2019).

Es interesante destacar que en las entrevistas y grupos focales los escraches a estudiantes no fueron valorados por los jóvenes como formas de resolver situaciones de acoso, sobre todo por su carácter individualizador y estigmatizador para el acusado. En este sentido, notamos un cambio reciente, sin duda producto de las últimas discusiones dentro del feminismo, respecto de los últimos años acerca de la percepción sobre este tipo de acciones como modos punitivistas de solución en los que se dejaba expuesto a quien realizaba una acción que incomodaba a otros u otras:

Para hacer que alguien deje de hacer algo, lo peor que podés hacer es que lo deje de hacer por miedo porque eso termina explotando de alguna otra forma [...] la escuela tiene que brindar espacios en los que los chicos se sientan contenidos y contenidas [...] una víctima siempre es una víctima pero ahí [refiriéndose al escrache] lo que termina pasando es que se terminan convirtiendo en víctimas los dos en un lugar donde hay una sola víctima, la marginación social no logra que alguien deje de hacer las cosas ni que cambies tu forma de pensar porque vos lo dejás de hacer por el miedo de lo que pueda pasar, por el miedo de que suban tu nombre a Instagram no porque realmente entendiste que estaba mal (estudiante varón, 5to. año, escuela pública universitaria). 
En el último tiempo, en Argentina y en otros países de América Latina ha cobrado lugar dentro del feminismo las discusiones sobre el punitivismo como respuesta inmediata y efectiva frente a situaciones de violencia. Dentro de las instituciones, estas formas se enmarcan en protocolos que establecen de manera directa la solución a diferentes tipos de situaciones, pero que apelan a formatos estandarizados y de rápida aplicación (Vázquez, Palumbo y Kohan, 2021). Segato se pregunta en una entrevista "¿qué es lo contrario a la impunidad? ¿El punitivismo?". Esta pregunta resulta pertinente para analizar los modos de sanción que se aplican en las escuelas (Alfie, 2018).

Esos abordajes nos permiten repensar también el sentido que le otorgan los estudiantes varones y mujeres a las sanciones. Tanto en los grupos focales como en las entrevistas formulamos la pregunta acerca de los aprendizajes que la escuela pretende transmitir mediante dichas instancias. Una estudiante que concurre a una escuela privada con orientación religiosa explica que las sanciones aplicadas no tienen mucho sentido para ella:

Cuando llegas a tres [firmas] tenés que quedarte una tarde después del colegio y te sientan en una clase y tenés que escribir un escrito que diga "Hice esto mal porque [...] y la consecuencia es...". Reflexionar por qué está mal lo que hiciste [...] las chicas que sé que tuvieron que quedarse después del colegio a hacer la reflexión decían “¿qué quieren que escriba?", no sé como que se sientan y guitarrean y escriben lo que se les ocurre y listo y se fueron y ya está. Supongo que escribieron cosas con sentido, pero sin sentir (estudiante mujer, 5to. año., escuela privada religiosa).

Por otra parte, muchos y muchas estudiantes mencionan la falta de un marco común en la aplicación de las sanciones o describen rasgos de arbitrariedad e injusticia; una estudiante de una escuela de gestión privada reflexiona: "A veces hay pequeños favoritismos en las clases y no va a ser lo mismo si tal hace una cosa que si tal hiciera lo mismo y no se aplica el mismo tipo de reto. Hay profesores que capaz se toman como una gracia algo que hace un alumno y si lo hace otro no" (estudiante mujer, 5 to año, escuela privada laica). En este mismo sentido, un estudiante de una escuela privada laica sostiene: "Termina dependiendo mucho del preceptor, te puede tocar uno muy apegado al reglamento o que por ahí lo sea por el lado del uniforme o que el uniforme no le importe pero que le importen otro tipo de cosas" (estudiante varón, 4to año, escuela privada laica).

Así también comenta con un poco de enojo y otro de resignación una estudiante de una escuela dependiente de la universidad: "A mí me sancionaron por no entregar en tiempo y forma el boletín, hay ciertas cosas tan absurdas por las que te sancionan, yo estaba en medio de una mudanza y qué sé yo dónde estaba el boletín" (estudiante mujer, 5 to año, escuela pública universitaria).

Como hemos mencionado, suspensiones y firmas -sea en formato de nota de cargo, amonestación o apercibimiento escrito- son las formas más comunes mencionadas por el estudiantado como modo de marcar límites a las transgresiones a las normas de convivencia. Frente a estas, destacan el diálogo como la mejor manera de intervenir y valorar su impronta pedagógica, sobre todo ante la reiteración de una falta o cuando dan cuenta de una situación problemática del estudiante que excede lo coyuntural. Un estudiante así lo señala: 
Son problemas mucho más profundos que llevó alcohol o se rateó [no ingresar a la escuela o retirarse antes sin permiso], tiene como un trasfondo que estaría bueno poder dialogarlo, poder hablarlo, contenerlo de cierto modo. Estaría bueno que se abra un canal de diálogo con los chicos cuando suceden estas situaciones (estudiante varón, 5to. año, escuela pública técnica).

Por su parte, otro estudiante manifiesta el valor del diálogo y señala el modo de proceder de una autoridad: "Tenés al regente del turno noche que en vez de cagarte a pedos y mandarte a tu casa, se sienta a hablar con vos cinco horas seguidas si es necesario" (estudiante varón, 5to. año, escuela pública universitaria).

Conviven en las escuelas un enfoque retribucionista y otro utilitarista (Zerbino, 2007) que se materializan tanto en lo discursivo simbólico como desde la aplicación de sanciones o las sugerencias que propone el consejo de convivencia. Marí (1983), siguiendo el discurso foucaultiano sobre el poder, introduce las condiciones de esta coexistencia no absolutista de ambos enfoques:

No hay que imaginar un discurso utilitarista aceptado y otro retribucionista excluido -o a la recíproca- que estuviesen insertos siempre en la misma estrategia. Hay que restituirlos con lo que acarrean de cosas dichas y ocultas, de enunciaciones requeridas, complementarias o prohibidas, con las variantes y los cambios que suponen quién habla, su inscripción en el poder y el contexto institucional en el que se halle colocado el portador del discurso (p. 86).

En la normativa también conviven estos dos enfoques que muchas veces desorientan a las escuelas: por una parte, se privilegian las ideas de prevención y reparación y se ajustan a una mirada retribucionista de las sanciones, al abrir un espacio para el diálogo y la reflexión conjunta frente al conflicto, y pretender que toda sanción tenga una finalidad educativa que, además, guarde relación con la falta. La prevención se entiende como la expectativa de anticipar el desarrollo de situaciones que conlleven algún tipo de conflicto e intervenir sobre ellas de forma previa, y la reparación, como la sanción que posibilita volver las cosas, lo más posible, a su situación anterior y que, además, habilita la responsabilización por parte del estudiantado, lo que genera un hecho formativo.

Por otra parte, las sanciones que propone la normativa y que se aplican con mayor frecuencia en las escuelas se alejan de la posibilidad de una intervención pedagógica que abone al aprendizaje y la reparación de la falta. Apercibimiento oral y escrito, cambio de división o de turno, diferentes tipos de separación transitoria y definitiva, así como acciones de reparación, componen la lista de sanciones mencionadas en la ley. Este abanico de medidas posibles, aunque no constituyan un techo para las escuelas, sino un piso para pensar otras opciones, dejan algún tipo de vacío y no proveen a las instituciones de otras instancias que puedan pensar las sanciones desde una concepción basada en la reciprocidad más que en la expiación (Piaget, 1932/1971).

A pesar de que la normativa propone un abordaje pedagógico del conflicto en el que se garantice la escucha de las distintas partes involucradas, y a través de mecanismos de participación colegiada y colectiva que conduzcan a la resolución más justa, en muchas ocasiones, los jóvenes encuentran las sanciones arbitrarias, injustas y discrecionales. 
Ahí es donde se soslaya la aplicación de la ley y sus variaciones en cada escuela. Lo que Bourdieu (2000) sostiene respecto a la aplicación de normativa jurídica resulta útil para pensar la puesta en práctica de las normas:

Aunque la existencia de reglas escritas tiende, indudablemente, a reducir la variabilidad de los comportamientos, las conductas de los agentes jurídicos pueden referirse y plegarse más estrictamente a las exigencias de la ley y queda siempre una parte de arbitrariedad en las decisiones judiciales, imputables a variables organizativas como la composición del grupo decisorio o las cualidades de los justiciables (p. 184).

\section{LA CONVIVENCIA ESCOLAR DURANTE LA EDUCACIÓN A DISTANCIA}

La educación a distancia irrumpió en la escuela como medio de enseñanza-aprendizaje a partir del aislamiento social preventivo y obligatorio producto de la pandemia por la COVID-19. Ingresó en la escena escolar y propició nuevas formas de enseñar, aprender y vincularse. Surgen así preguntas inéditas en un contexto novedoso. Si el sistema de convivencia tiene un espacio de injerencia dentro del edificio escolar, ¿cuál es el adentro y el afuera de la escuela durante la virtualidad?, ¿cuáles son los nuevos conflictos que han aparecido?, ¿cómo se han resuelto?, ¿cómo se reconfiguraron los vínculos entre estudiantes y con los docentes? y ¿cuáles son las normas que rigen y tienen lugar?

Por su parte, los estudiantes relataron en las entrevistas y grupos focales situaciones que nos permiten sumar reflexiones respecto de estos cuestionamientos. $\mathrm{Al}$ igual que en la modalidad presencial, las normas no son comunes en todos los establecimientos y, al mismo tiempo, se acrecentó la disparidad de criterios entre docentes de una misma escuela. Quizá la ausencia del espacio físico escolar y la presencia de las casas ocasionó que operen con más fuerza los criterios individuales por encima de los institucionales. Además, a las reglas de comportamiento sobre cómo estar/habitar las escuelas, se sumaron nuevas pautas u otras cobraron más peso: asistir a los encuentros sincrónicos, prender las cámaras, silenciarse y participar en los encuentros.

Los conflictos relacionados con cuestiones académicas fueron mencionados también como los más recurrentes durante la virtualidad y se refirieron a la entrega de trabajo en determinadas fechas, enviar varios o varias el mismo trabajo y la sobreexigencia que se dio por el método de enseñanza-aprendizaje que consistía, en muchos casos, en realizar y subir trabajos a la plataforma utilizada, pero sin una devolución. Un estudiante pone de manifiesto esta situación:

Conflictos con profesores que nos pidieron catorce trabajos y nunca nos corrigieron y que quizás cuando quisimos ponernos en contacto con ellos se enojaron un poquito y tampoco tenemos preceptores que colaboren con esta situación, no son muy proactivos por eso estamos averiguando el mail de los directivos porque yo me comprometo, entrego todo en tiempo y forma pero también tengo que tener una devolución sino cómo voy a saber recién dentro de seis meses si me fue bien o me fue mal y ese fue un conflicto (estudiante varón, 6to. año, escuela pública técnica).

Otros dos estudiantes de una escuela privada comentan también la falta de criterios claros acerca de las demandas académicas: 
Estudiante 1: una profesora nos dio un trabajo para hacer..., pero nunca explicó lo que íbamos a hacer en clase, al día siguiente nos unimos al Meet y nos dijo "Les doy estas preguntas para responder en cuarenta minutos en parejas" y entre una cosa y la otra nos quedaban solo quince minutos para hacer todo el trabajo completo y discutimos con la profesora. Todo de manera respetuosa, pero parece que interpretó mal a un compañero que le dijo algo (estudiante varón, 5to año, escuela privada laica).

Estudiante 2: ese compañero a quien le terminó poniendo una nota de disciplina había entrado como veinte minutos más tarde y ella le dice "no te podés unir ahora, me estás tomando el pelo. Te voy a poner ausente" (estudiante mujer, 5to año, escuela privada laica).

Parte de la disparidad en la exigencia, o no, de determinadas normas en la virtualidad estuvo condicionada por las posibilidades tecnológicas de la matrícula de las escuelas. En algunas escuelas privadas o estatales que atienden a sectores socioeconómicos medios o altos pudieron desarrollar clases sincrónicas emulando las presenciales y exigir la participación de los estudiantes en ellas. En estos casos, destaca que, en algunas ocasiones, hubo profesores que propusieron actividades de producción de trabajos en grupos más interesantes, como un podcast, un video, un programa de radio y que, además, se trabajaron los contenidos curriculares para analizar el contexto: "En muchas materias nos pidieron hacer un video o un flyer y era algo que tal vez en la presencialidad terminaba en un oral" (estudiante mujer, 5to año, escuela estatal universitaria).

Esto fue más dificultoso en escuelas donde la falta de conexión o de dispositivos o la mayor desigualdad en dicho acceso impedía participar de estos encuentros. En un grupo focal, un estudiante narra la solución que encontraron algunos docentes para lograr sostener la escolaridad virtual:

De mi curso somos catorce de los cuales nos conectamos tres, muchos profesores al ver que éramos pocos los que nos conectábamos comenzaron a organizar pequeños recreos virtuales sin nota para que compartiéramos memes de la materia por ejemplo o compartiendo distintas actividades y así poder tener una conexión con el colegio (estudiante varón, 5to. año, escuela estatal bachiller).

Por otra parte, una estudiante da cuenta de la "materialidad" de muchas normas de convivencia de la escuela que se han perdido en un medio virtual:

La mayoría de las normas son cosas materiales como la vestimenta o llegar tarde, cosas que se hacen en la presencialidad, pero en la virtualidad no hubo eso, mucho menos en los zooms: prender la cámara también dependía del profesor, no nos obligaban, pero, por ejemplo, un profesor mío tuvo un inconveniente y nos empezó a decir que prendamos la cámara (estudiante mujer, 4to. año, escuela privada laica).

Así como han aparecido conflictos nuevos, los estudiantes han relatado nuevas configuraciones en los vínculos entre ellos. Según referencian, a la escuela le costó trabajar en la vinculación en la distancia y esto llevó a que, en muchos casos, fueran los propios estudiantes los que se acompañaran entre ellos y ellas. En varias ocasiones, se generó más solidaridad, se pasaban trabajos y ayudaban a quienes no tenían acceso a tecnología acorde para realizar y entregar lo solicitado. Una estudiante relata:

Ahora en el contexto de la pandemia en mi clase nos estamos ayudando mucho, si le preguntan algo a alguien que no sabe nos lo pasamos por el grupo [refiriéndose al grupo de WhatsApp de los y las compañeros del curso] o nos pasamos la tarea. Nos estamos ayudando por ahí y te hace sentir bastante bien que alguien te pueda ayudar (estudiante mujer, 5to. año, escuela privada laica). 
La siguiente cita permite comprender el modo en que el vínculo pedagógico con muchos docentes se ha debilitado al tener menos encuentros en la virtualidad, mientras que la relación entre estudiantes ha logrado fortalecerse:

Lo que más se debilitó es el vínculo pedagógico con el docente, si bien tenemos reuniones y hablamos y nos preguntan cómo estamos, no es lo mismo que en la presencialidad. Hay complicaciones para comunicarnos con los docentes y en ese sentido fue un fortalecimiento de los vínculos entre nosotros. El año pasado en la presencialidad estábamos muy metidos en lo que es nuestro grupo de personas íntimas como que este año si bien no es que somos todos mejores amigos sí estamos teniendo más compañerismo [...] en nuestra escuela se está dando que tenemos un montón de trabajos prácticos, en algunas materias tenemos más de veinte y se está dando más compañerismo, nos pasamos los trabajos o si no entendemos algo nos ayudamos y es algo que en la presencialidad no pasaba. También hubo una situación de descarga, de contención con que quizás antes no nos llevábamos tanto, que en la presencialidad eso se daba más con los profesores y ahora no estaba y supimos entre compañeros generar esas redes. Con los profesores si bien la relación es buena no es muy fluida como antes (estudiante mujer, 5to. año, escuela pública bachiller).

Los estudiantes varones y mujeres parecieran haber encontrado en las redes y las plataformas de comunicación un espacio de encuentro y contención que, tal vez, en la modalidad presencial no era promovido. No solo los cursos encontraron en los medios virtuales una forma de sostén y acompañamiento, sino que en aquellas escuelas donde hay centro de estudiantes las actividades propuestas a través de las redes sociales han sido también un anclaje con la escuela y una instancia de contención que produce vínculos entre el estudiantado.

A pesar de la solidaridad generada, el encuentro y el contacto físico con amigos y amigas en la presencialidad es de las cuestiones que más extrañan. En la encuesta, el 64\% del alumnado mencionó el pasar tiempo con compañeros como lo que más extrañan junto con los abrazos, cuya respuesta obtuvo un 46\%. Esta opción obtuvo una diferencia porcentual entre las respuestas de mujeres (50\%) y de varones (35\%). Esta distancia de 15 puntos puede ser leída desde los estereotipos de género que habilitan para las mujeres mayores posibilidades de expresión afectiva, mientras que las restringen para los varones en tanto que esta puede ser interpretada como signo de debilidad.

A continuación, citamos algunas intervenciones de las entrevistas y grupos focales respecto de lo que más extrañan: "Estar en presencia de otras personas, estar juntos, pasarlo bien” (estudiante varón, 5to. año, escuela privada técnica); "estar con amigos, los recreos, comer juntos" (estudiante varón, 5to. año, escuela privada laica); "extraño compartir el lugar, saber lo que le pasó a cada uno" (estudiante mujer, 4to. año, escuela privada laica), “... mis amigos del colegio son lo más cercano que tengo y si no hubiese tenido clases presenciales no los conocería y eso es renecesario, sociabilizar y eso solo puede darse dentro de un lugar donde estén todos" (estudiante mujer, 5to. año, escuela estatal universitaria).

Como vimos, la convivencia implica desarrollar maneras de estar con otros, de convivir en la escuela, reconocer la existencia de los conflictos para encontrar formas de resolución y buscar una construcción de ciudadanía juvenil basada en mecanismos democráticos y marcos comunes de justicia entre estudiantes y docentes (Núñez, 2013). Ello permite enmarcar la convivencia en una cosmovisión acerca de la vida en común en la escuela que excede la intención de pensarla de modo instrumental solo 
como forma de "atenuar" o "eliminar" los conflictos, pero que, muchas veces, es la menos trabajada en la escuela.

Así, podemos tomar dos dimensiones de la convivencia escolar que desarrollan Viscardi y Alonso (2013) a partir de una investigación realizada en Uruguay. Por una parte, la convivencia constituye el conjunto de principios que guían las prácticas y define los vínculos al interior de las escuelas, donde "se conforma, allí, una gramática de la convivencia al enunciarse su sistema de reglas y normas" (p. 29). Por otra, aparece otra dimensión más ligada al estado de las relaciones interpersonales que suceden en la cotidianidad escolar y que conforman las diferentes culturas escolares. Convivencia es definida por estos autores como la reflexión sobre las reglas para "estar con otros" y muestra el "estado real" de la convivencia. Durante la educación virtual, los estudiantes varones y mujeres han logrado ubicarse desde esta concepción de la convivencia, al revisar y fortalecer los vínculos entre ellos y ellas de un modo solidario.

\section{CONCLUSIONES}

La convivencia escolar, en cuanto perspectiva y sistema instituido, constituye una manera particular de socialización, formación ciudadana, así como un nuevo modo de gobierno institucional para la gestión de los conflictos. En el ámbito educativo debería adquirir, fundamentalmente, dos sentidos: un nuevo vínculo entre adultos, adultas y jóvenes con base en la construcción consensuada de normas y participación de ambos en la resolución de conflictos, y una nueva forma de socialización política del estudiantado en la escuela como espacio de experiencias sobre la participación y las prácticas democráticas.

Luego de este recorrido, notamos que, si bien el sistema de convivencia se encuentra mucho más extendido y la gran mayoría de los establecimientos educativos de las distintas gestiones cuentan con un reglamento que contiene las normas que rigen la cotidianidad escolar, el desafío que aún queda pendiente es la inclusión del alumnado en el proceso de su elaboración, de modo que las normas puedan apropiarse y resulten significativas para ellos y ellas.

Ante la situación vivida en el contexto de pandemia, resulta adecuado preguntarse por la pertinencia y vigencia de una normativa de convivencia promulgada hace ya más de dos décadas. El análisis realizado pone de manifiesto la necesidad de extender y potenciar las instancias de deliberación colectiva y posibilita visualizar la importancia de actualizar los acuerdos de convivencia en función de las características propias del contexto y las particularidades de las distintas situaciones.

En estos puntos hallamos que los objetos, principios y objetivos que refieren la promoción de prácticas democráticas que posibiliten el cumplimiento de los fines educativos de cada escuela (Ciudad de Buenos Aires, 1999, artículos 2oㅗ $5^{\circ}$ y 6을 no entran en pausa cuando la modalidad es a distancia; muy por el contrario, deben profundizarse buscando generar espacios de escucha y participación en este contexto inédito e incierto, y aplicar las adecuaciones necesarias que permitan orientar las formas de vinculación de los distintos actores escolares. A la vez, es necesario que las escuelas puedan repensar las normas propias y trascender aspectos concretos de la vestimenta y la puntualidad para poder dar lugar a normas que promuevan el mejor modo de encuentro con otros y otras. 
Es un paso fundamental que el estudiantado encuentre espacios de participación que los impliquen en la vida escolar, porque les ha permitido avanzar en la democratización de las relaciones dentro de las escuelas. Esto se ha conseguido con mayor o menor éxito en las diferentes escuelas. Aquellas que han avanzado lograron generar lo que Fernández (2012) denomina convivencia, no solo como una serie de reglas escritas, sino también la convivencia escolar que reconoce al otro como miembro de una misma comunidad. Retomando lo expresado, esto es posible si ambos se rigen por las mismas normas y, sobre todo, si son portadores de los mismos derechos y obligaciones, teniendo en cuenta las diferencias entre docentes y estudiantes en relación con las responsabilidades y roles que le competen a cada uno.

En el caso de las sanciones, hallamos una tensión, difícil de resolver, entre la búsqueda de un marco común para evitar que la resolución de conflictos adquiera características arbitrarias o injustas, pero que no resulte ser aplicada desde un modo punitivo que no tiene en cuenta la complejidad y el contexto de la situación específica. Los estudiantes se inclinan por un modo dialógico como forma pedagógica de intervención escolar frente a las faltas, sobre todo en las vinculadas a consumos problemáticos, o la reiteración de transgresiones o situaciones de acoso entre estudiantes.

Durante el aislamiento, que implicó el sostenimiento de la escolaridad a distancia, los conflictos más recurrentes tuvieron que ver con las exigencias académicas y, en menor medida, con las faltas más comunes en la presencialidad, como el cumplimiento del horario, la vestimenta, entre otras. En este sentido, cobraron más relevancia algunas problemáticas y otras quedaron relegadas, como los conflictos entre pares que, por el contrario, en muchos casos, los vínculos entre estudiantes se vieron fortalecidos. La convivencia logra, así, ir más allá del trabajo en torno al conflicto y lo sancionatorio para abordar otros ejes concernientes a la solidaridad, la empatía y el compañerismo. La escuela presencial tiene como desafío retomar este aspecto y fortalecerlo.

\section{REFERENCIAS BIBLIOGRÁFICAS}

Alfie, C. (2018). Rita Segato: "El feminismo punitivista puede hacer caer por tierra una gran cantidad de conquistas". APU. Agencia Paco Urondo. Periodismo Militante. https://www.agenciapacourondo.com.ar/generos/rita-segato-elfeminismo-punitivista-puede-hacer-caer-por-tierra-una-gran-cantidad-de)

Berger, P. y Luckmann, T. (1986). La construcción social de la realidad. Buenos Aires: Amorrortu.

Bourdieu, P. (2000). La fuerza del derecho. Elementos para una sociología del campo jurídico. En Poder, derecho y clases sociales (pp. 165-224). Bilbao, España: Editorial Palimpesto.

Ciudad de Buenos Aires (1999). Ley № 223 de 1999 (Ley de Convivencia Escolar).

https://www.buenosaires.gob.ar/sites/gcaba/files/ley_223_sistema_escolar_ de_convivencia_-.pdf

Dussel, I. (2005). Sobre la dificultad de construir consensos en educación. Una mirada desde la cultura política y la cultura de gestión en las políticas educativas. En Pactos y compromisos en las políticas educativas en la Argentina. Madrid, España: Fundación Santillana Tedesco. 
Faur, E. (2019). Del escrache a la pedagogía del deseo. Revista Anfibia. http:// revistaanfibia.com/cronica/del-escrache-la-pedagogia-del-deseo/

Fernández Enguita, M. (2012). Escuela y ciudadanía en la era global. En G. Diker y G. Frigerio (comps.). Educar: posiciones acerca de lo común (pp. 15-30). Buenos Aires, Argentina: Del Estante.

Fridman, D. (2014). El sistema escolar de convivencia: una mirada desde su sanción hasta su implementación actual en la Ciudad de Buenos Aires. Revista Irice, núm. 27, pp. 123-149.

Fridman, D. y Litichever, L. (2016). Consejos de convivencia. Un análisis de las formas de sanción escolar. Revista Interdisciplinaria de Reflexión y Experiencia Educativa Convocación, año VI, diciembre, pp. 15-25.

González del Cerro, C. (2017). Del \#NiUnaMenos a la regulación de la vestimenta escolar. Nuevos estilos de participación política juvenil. En V. Orce (comp.). La educación como espacio de disputa: miradas y experiencias de los/las investigadores/as en formación (pp. 63-86). CABA, Argentina: Editorial de la Facultad de Filosofía y Letras Universidad de Buenos Aires.

Jares, X. (1997). El lugar del conflicto en la organización escolar. Revista Iberoamericana de Educación, núm. 15. http://www.oei.es/oeivirt/rie15a02. htm

Kriger, M. y Dukuen, J. (2017). Haciendo de la necesidad virtud: socialización política y herencia familiar entre becarias de un colegio de clases altas (CABA). Revista Pilquen, vol. 20, núm. 3. file:///Users/denisefridman/Downloads/ Dialnet-HaciendoDeLaNecesidadVirtud-6128189.pdf

Litichever, L. (2019). Dinámicas de la convivencia: nuevos modos de resolver los conflictos en las escuelas. En P. Núnez, L. Litichever y D. L. Fridman (comps.). Escuela secundaria, convivencia y participación. Colección Educación y Sociedad 05-Eudeba-OEI.

Litichever, L. (2012). La discrecionalidad en la aplicación de sanciones en la escuela secundaria. Miradas diferentes de docentes y estudiantes. Ponencia presentada en las I Jornadas de Jóvenes Investigadores en Educación. FlacsoArgentina, 12, 13 y 14 de septiembre. http://editorial.flacso.org.ar/jornadas.

Litichever, L., Machado, L., Núñez, P., Roldán, S. y Stagno, L. (2008). Nuevas y viejas regulaciones: un análisis de los reglamentos de convivencia en la escuela media. Revista Última Década. CIDPA, año 16, núm. 28, julio, pp. 93-121. http://www.scielo.cl/scielo.php?script=sci_arttext\&pid=S0718$22362008000100006 \& \operatorname{lng}=e s \& n r m=i s o \& \operatorname{tlng}=e s$

Marí, E. (1983). La problemática del castigo. El discurso de Jeremy Bentham y Michel Foucault. Buenos Aires, Argentina: Hachette.

Ministerio de Educación (2020). Anuario Estadístico Educativo 2019. https:// www.argentina.gob.ar/educacion/evaluacion-informacion-educativa/anuarios

Mouffe, Ch. (2005). Políticas y pasiones: las apuestas de la democracia. En L. Arfuch (comp.). Pensar este tiempo. Espacios, afectos, pertenencia. Buenos Aires, Paidós.

Núñez, P. (2013). La política en la escuela. Buenos Aires, Argentina: La Crujía Ediciones.

Paulín, H. (2002). Los consejos de convivencia: ¿una alternativa democratizadora de la escuela o más de lo mismo? Educar, núm. 20, pp. 36-47. 
Piaget, J. (1932/1971). El criterio moral en el niño. Barcelona, España: Fontanella.

Rockwell, E. (2009). La experiencia etnográfica. Buenos Aires: Paidós.

Siede, I. (2007). La educación política. Ensayo sobre ética y ciudadanía en la escuela. Buenos Aires, Argentina: Paidós.

Simmel, G. (1908/ 2010). El conflicto. Sociología del antagonismo. Madrid, España: Sequitur.

Southwell, M. (comp.) (2020). Hacer posible la escuela. Vínculos generacionales en la secundaria. Buenos Aires, Argentina: UNIPE Editorial. https://editorial.unipe.edu.ar/colecciones/investigaciones/hacer-posible-la-escuelav\%C3\%ADnculos-generacionales-en-la-secundaria-detail?fbclid=IwAR1Qu Q4quTukT1JNyxMb2VAxKpnZ8qmw0gM6x6BBba0Hu2xH7dWUGNtgykc

Tenti Fanfani, E. (2009). Diversidad cultural y ciudadanía activa. Consideraciones sociológicas. En E. Tenti Fanfani (ed.). Diversidad cultural, desigualdad social y estrategias de políticas educativas (pp. 79-110). Buenos Aires, Argentina: IIPE Unesco.

Vázquez Laba, V., Palumbo, M. y Kohan, A. (2021). Por una emancipación singular. En D. Losiggio y M. Solana (comps.). Acciones y debates feministas en las universidades. Florencio Varela, Argentina: Editorial UNAJ.

Viscardi, N. (2008). Violencia en las aulas. Práctica educativa y conflicto escolar y exclusión social. En R. Paternain y R. Sanseviero (orgs.). Violencia, inseguridad y miedos en el Uruguay. ¿Qué tienen para decir las ciencias sociales? (pp. 143-158). Montevideo, Uruguay: FESUR.

Viscardi, N. y Alonso, N. (2013). Gramática (s) de la convivencia escolar. Un examen a la cotidianidad escolar y la cultura política en la educación primaria y media en Uruguay. Montevideo, Uruguay: Administración Nación de Educación Pública/ CODICEN.

Zerbino, M. (2007). La estética del castigo. En G. Frigerio y G. Diker (comps.). Educar: (sobre) impresiones estéticas (pp. 237-270). CEM Buenos Aires, Argentina: Del Estante Editorial, Serie Seminario. 